\title{
Epilepsie und Alkohol
}

Seit der Antike ist bekannt, dass ein erhöhter Alkoholkonsum das Auftreten epileptischer Anfälle begünstigt. Die neurobiologische Grundlage dieses Zusammenhangs ist komplex und bisher nicht in allen Details verstanden. Obwohl im neurologischen und psychiatrischen Klinikalltag alkoholbezogene epileptische Anfälle ein häufiges Problem darstellen, gibt es keine einheitlichen Richtlinien hinsichtlich des diagnostischen Managements und des therapeutischen Vorgehens. In den aktuellen Suchtleitlinien findet das Problem epileptischer Anfälle im Alkoholentzug nach akutem oder chronischem Konsum keine Berücksichtigung.

Ein nichtrisikoarmer Alkoholkonsum stellt vor allem in den Industrienationen ein weit verbreitetes Problem dar. So besteht in Deutschland bei etwa 1,6 Millionen Menschen eine Alkoholabhängigkeit, bei 2,7 Millionen ein Alkoholmissbrauch, d.h. ein schädlicher Gebrauch mit Beeinträchtigung der physischen und psychischen Gesundheit, und bei 5 Millionen ein riskanter Konsum, d.h. eine tägliche Alkoholeinnahme von 31 bis $60 \mathrm{~g}$ bei Männern und 21 bis $40 \mathrm{~g}$ bei Frauen. Eine abrupte Alkoholkarenz bei zuvor chronischem Konsum führt bei vielen Patienten über mehrere Tage zu mannigfaltigen psychiatrischen und vegetativen Symptomen. Im Rahmen des Alkoholentzugssyndroms treten bei etwa 8 bis $10 \%$ der Patienten epileptische Anfälle auf. Die meisten dieser Symptome sind Folge einer alkoholbedingten Dysbalance von inhibitorischen und exzitatorischen Neurotransmittern und der entsprechenden Rezeptoraktivität. Eine pharmakologische Verstärkung der neuronalen Inhibition lindert die meisten Symptome des Alkoholentzugssyndroms und die Wahrscheinlichkeit epileptischer Anfälle wird signifikant reduziert.

Bei Patienten mit einer Epilepsie finden sich Alkoholabhängigkeit und -missbrauch sowie ein riskanter Konsum nicht seltener als in der Ge- samtbevölkerung. Diese häufige Komorbidität und ihre Folgeerscheinungen stellen die antiepileptische Therapie vor dem Hintergrund pharmakodynamischer und -kinetischer Besonderheiten vor ganz spezifische Herausforderungen. Primär neuronal inhibitorisch wirkende Antiepileptika können in ihrer Effizienz bei reduzierter Rezeptoraktivität beeinträchtigt sein. Leichte und schwere Leberfunktionsstörungen bei erhöhtem oder chronischem Alkoholkonsum bedingen eine gesteigerte oder auch deutlich reduzierte hepatische Metabolisierung lebergängiger Antiepileptika. Die Folge sind zum Teil drastische Änderungen der Serumkonzentrationen der antiepileptischen Substanzen, die entsprechende Dosisanpassungen in Abhängigkeit vom Stadium der Leberschädigung erforderlich machen. Neuere Antiepileptika mit einer geringen hepatischen Verstoffwechselung haben hier in der Regel günstigere pharmakokinetische Eigenschaften als die konventionellen Substanzen.

Ein akut erhöhter Alkoholkonsum gepaart mit Schlafentzug ist häufig ein Trigger - nicht die Ursache - für die Erstmanifestation einer Epilepsie. Gerade bei jugendlichen Patienten mit einer idiopathisch generalisierten Epilepsie führt diese Konstellation mitunter zum Auftreten eines ersten epileptischen Anfalls.

Letztendlich bleibt die Frage, ob Patienten mit Epilepsie generell von Alkoholkonsum abgeraten werden sollte. Die vorhandenen Daten geben jedoch keinen ausreichenden Anhalt, dass sich ein maßvoller Alkoholkonsum negativ auf die Epilepsie auswirken kann. So lässt sich bei den meisten Patienten mit Epilepsie eine weitere Stigmatisierung vermeiden.

In diesem Schwerpunktheft werden unterschiedliche Aspekte des Zusammenhangs von epileptischen Anfällen und Epilepsie sowie akutem und chronischem Alkoholkonsum aus neurologisch-epileptologischer und psychiatrischer Perspektive diskutiert.

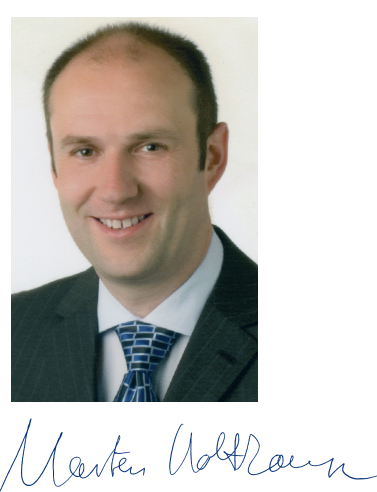

PD Dr. Martin Holtkamp, Berlin 